$5-15-2009$

\title{
Reductive Decomposition of A Diazonium Intermediate by Dithiothreitol Affects The Determination of NOS Turnover Rates
}

Perera N. Indika

Cleveland State University

Talal Sabbagh

Cleveland State University

Jean A. Boutros

Cleveland State University

Mekki Bayachou

Cleveland State University, M.BAYACHOU@csuohio.edu

Follow this and additional works at: https://engagedscholarship.csuohio.edu/scichem_facpub

Part of the Chemistry Commons

How does access to this work benefit you? Let us know!

\section{Recommended Citation}

Indika, Perera N.; Sabbagh, Talal; Boutros, Jean A.; and Bayachou, Mekki, "Reductive Decomposition of A Diazonium Intermediate by Dithiothreitol Affects The Determination of NOS Turnover Rates" (2009). Chemistry Faculty Publications. 327.

https://engagedscholarship.csuohio.edu/scichem_facpub/327

This Article is brought to you for free and open access by the Chemistry Department at EngagedScholarship@CSU. It has been accepted for inclusion in Chemistry Faculty Publications by an authorized administrator of EngagedScholarship@CSU. For more information, please contact library.es@csuohio.edu. 


\title{
Reductive decomposition of a diazonium intermediate by dithiothreitol affects the determination of NOS turnover rates
}

\author{
Indika N. Perera, Talal Sabbagh, Jean A. Boutros, Mekki Bayachou
}

\section{Introduction}

Determination of trace amounts of nitrite is crucial in a number of mechanistic and kinetic investigations addressing structure-function of enzymes. Examples include investigations addressing mechanisms and kinetics of the molecular function of enzymes such as Nitrite Reductases (NiR) [1] and nitric oxide synthases (NOS) [2]. In the latter case, accurate determination of nitrite $\left(\mathrm{NO}_{2}{ }^{-}\right)$as a breakdown product of nitric oxide (NO) is of particular importance in current investigations, which aim to understand the molecular function of NOS enzymes. Nitric oxide synthases are heme enzymes that catalyze the in vivo synthesis of nitric oxide, a diatomic molecule that was found to mediate numerous physiological processes and is involved in the development of a host of pathological states [3]. NO is involved in vasodilation, neurotransmission, cytotoxicity, and cytoprotective processes $[4,5]$. It is biosynthesized by enzymatic oxidation of one terminal guanidine-nitrogen of the amino acid L-arginine through the $\mathrm{N}$-hydroxy-L-arginine intermediate, yielding L-citrulline as a coproduct (Scheme 1).

Although several methods have been developed to determine the NOS turnovers [6-8], NO is often quantified in the form of nitrite $\left(\mathrm{NO}_{2}^{-}\right)$, a stable breakdown product of $\mathrm{NO}$ in aerobic reaction media. Various methods are employed to quantify nitrite accurately especially in biological samples [9-13]. The most commonly used technique to quantify nitrite in NOS mechanistic investigations is using a spectrophotometric assay based on the Griess reaction through a standard calibration curve $[14,15]$. This assay is based on a two-step diazotization reaction in which acidified nitrite produces a nitrosating agent which reacts with sulfanilamide to produce the diazonium derivative. The diazonium ion is then coupled to $\mathrm{N}$-1-napthylethylenediamine dihydrochloride (NED) to stoichiometrically form the final azo-product which absorbs at $540 \mathrm{~nm}$ (Supporting information, Scheme S1). The Griess assay is widely used to determine nitrite in a variety of biological and experimental matrices such as plasma, serum, urine, and tissue culture media [16-18]. However, serious accuracy problems may arise in the presence of interfering agents, especially if these are not taken into account during the external calibration. It has been already reported that the NOS enzyme itself spectrally interferes with the final product of Griess assay [19]. The NADPH, an essential cofactor in NOS reaction, is also a known interferent in the Griess assay [20]. John Moody and Shaw recently published a reevaluation study of the Griess assay in terms of the extent of interference brought by nicotinamide nucleotides in the assay medium [21]. A prior review by Fox and Suhre documented interferences by a number of agents including thiols [22].

Accurate quantification of nitrite is critical for reliable determination of NOS turnover and other kinetic aspects; it thus can affect 


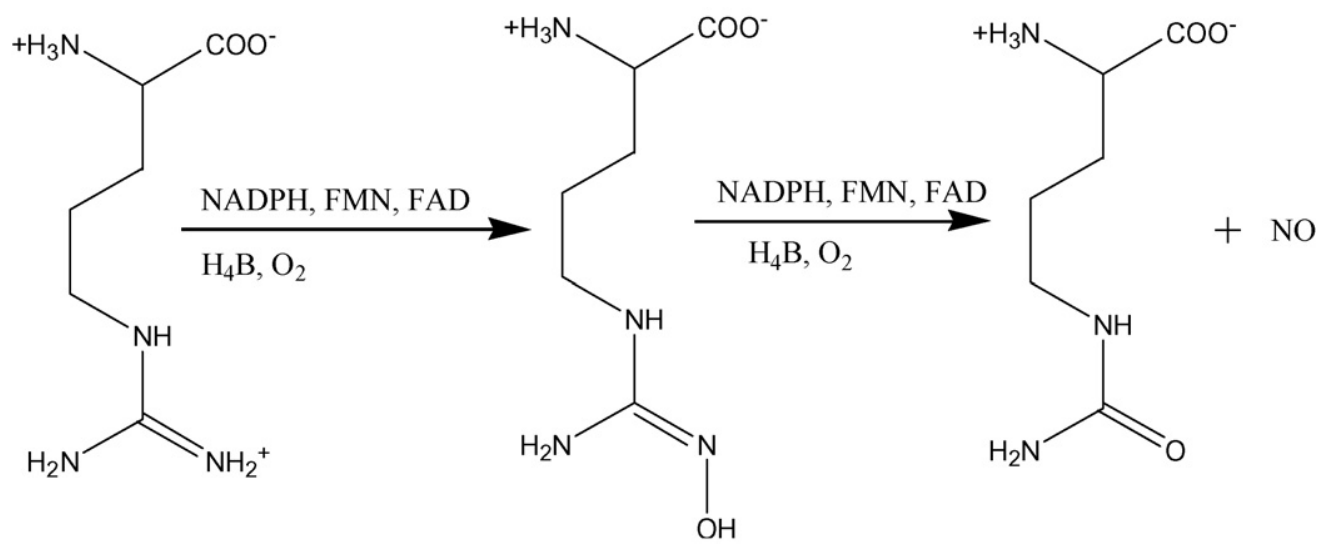

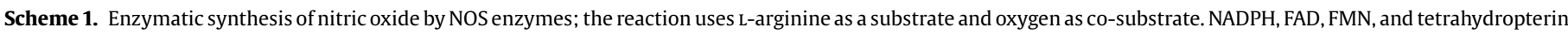
are also necessary for the NOS function.

all derived mechanistic/kinetic interpretations. The cationic diazonium intermediate that forms during the first step of the Griess assay is vulnerable towards most reducing agents in the assay medium, especially dithiothreitol (DTT), which is widely used in NOS reactions. The interaction between diazonium intermediate and DTT, especially when present in high concentrations $0.5 \mathrm{mM}$ or higher), results in a critically low yield for final azo-compound, which seriously affects the sensitivity of the method. This, in turn, leads to serious limitations for nitrite determination especially in the low concentration range (i.e. $2.5-10 \mu \mathrm{M}$ ), where important mechanistic information is usually derived through measurement of initial rates.

In this work, we probe the nature of critical interaction of the diazonium intermediate with DTT as a reducing agent using electrochemical and spectrophotometric techniques. The study also explores how DTT concentration affects the sensitivity of the Griess assay for nitrite determination, even when matrix correction (i.e. using DTT in standards) is used in the external calibration method. The study further investigates possibilities to minimize the matrix difference to achieve high accuracy and sensitivity using standard addition method.

\section{Experimental}

\section{Reagents}

All the chemicals used were of analytical grade. Nanopure deionized water ( specific resistance $>18.2 \Omega \mathrm{cm}$ ) used in all experiments was supplied by a Barnstead water purification system. All working solutions of nitrite were prepared using $0.1 \mathrm{M}$ standard nitrite solution. Dithiothreitol and NADPH were purchased from Sigma. The Griess reagent kit was purchased from Promega (Madison, WI) and was used following the technical instructions provided. Briefly, the analyte solution is incubated with sulfanilamide first for 10-15 min, followed by the addition of NED (10-15 $\mathrm{min})$, and the absorbance of the final azo dye $\left(\lambda_{\max }\right.$ at $\left.540 \mathrm{~nm}\right)$ is recorded. It is worth mentioning the concentration of the Griess ingredients in the stock solutions of the kit. Based on the chemical specifications on the Promega kit, the concentration of sulfanilamide solution is $58 \mathrm{mM}$ while the NED solution is at $3.9 \mathrm{mM}$. These concentrations ensure that the Griess assay ingredients are always in excess compared to the nitrite analyte.

\section{Apparatus}

UV-vis absorbance spectra were recorded on Agilent 8453 spectrophotometer. Spectra were collected between 400 and $650 \mathrm{~nm}$.
Cyclic voltammetry was performed in a standard three-electrode cell using a BAS100W electrochemical workstation (Bioanalytical Systems Inc.). A gold working electrode $\left(\mathrm{CHI}\right.$, Area $=0.0314 \mathrm{~cm}^{2}$ ) was polished with alumina slurry (successively with 0.3 and $0.05 \mu \mathrm{m}$ ), and cleaned in ultrasound bath in deionized water.

\section{Electrochemical measurements}

All electrochemical measurements were carried out in nitrogen purged $0.05 \mathrm{M} \mathrm{NaCl}$ solution. The solutions were stirred between the cyclic voltammetric experiments; all potential are reported versus the $\mathrm{Ag} / \mathrm{AgCl}$ reference electrode. After each scan in solutions of diazonium ions, the working electrode was cleaned with piranha solution $\left(70 \% \mathrm{H}_{2} \mathrm{SO}_{4}\right.$ and $\left.30 \% \mathrm{H}_{2} \mathrm{O}_{2}\right)$ and polished as described above.

\section{Procedure for calibration curves}

The Griess assay used widely is a two-step process. First the analyte aliquot is incubated for $15 \mathrm{~min}$ with an excess sulfanilamide to generate the diazonium cation. NED (in excess of the diazonium cation formed) is then added to the assay medium and the color is allowed to develop. Absorbance is recorded after exactly 15 min of reaction time. Calibration curves were constructed for the range of 2.5-15 $\mu \mathrm{M}$ nitrite; standards were prepared using $0.1 \mathrm{M}$ nitrite stock solution. $500 \mu \mathrm{L}$-aliquots of standards are incubated for 15 min each with $100 \mu \mathrm{L}$ sulfanilamide and $100 \mu \mathrm{L}$ NED. Deionized water is added up to $1.00 \mathrm{~mL}$ and absorbance measurement taken at $540 \mathrm{~nm}$.

To evaluate the matrix effect, samples for recovery studies are prepared by mixing various amounts of nitrite and DTT (from $1 \mathrm{mM}$ working solutions) and diluting with deionized water to $1.00 \mathrm{~mL}$. $500 \mu \mathrm{L}$ aliquots of each unknown were incubated for $15 \mathrm{~min}$ with $100 \mu \mathrm{L}$ sulfanilamide followed by $100 \mu \mathrm{L}$ NED and diluted with deionized water to $1.00 \mathrm{~mL}$. The absorbance is then measured and the nitrite amount is quantified using a suitable calibration curve.

\section{Procedure for standard addition method}

Samples containing two different concentrations of nitrite (3 and $6 \mu \mathrm{M}$, prepared from a $0.1 \mathrm{M}$ stock solution just as we did for the regular calibration method) in the presence of three representative concentrations of DTT $(0.0,0.4$, and $1.0 \mathrm{mM})$ were prepared, giving a total of six test solutions. The total volume of each sample analyzed was $3.00 \mathrm{~mL}$. The range of nitrite concentration in these working samples was selected based on actual ranges used in NOS reaction investigations. Each test solution was divided into 
(A)

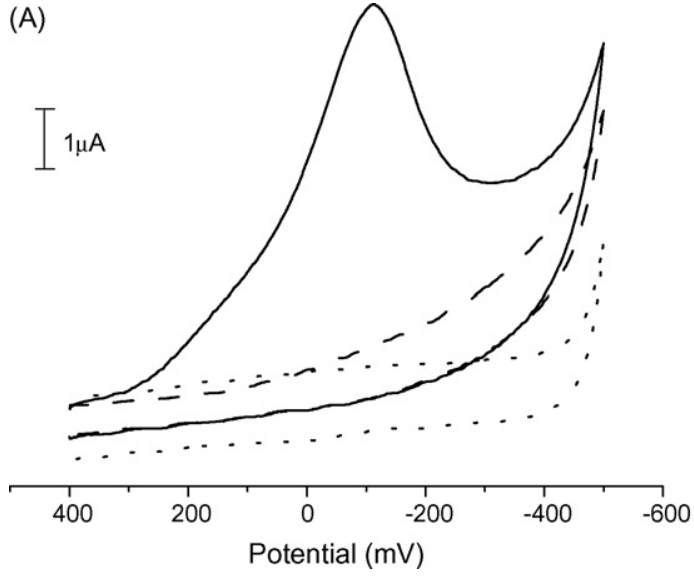

(B)

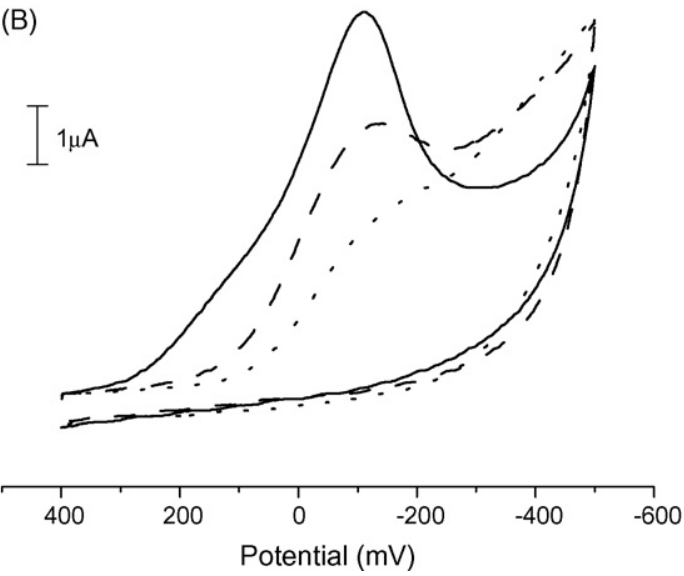

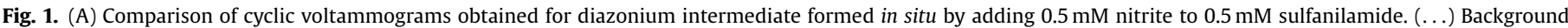

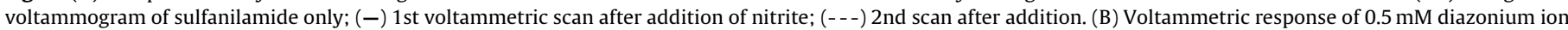
formed in situ in absence (-) and presence of $0.5 \mathrm{mM}$ DTT (-- ) and $1.0 \mathrm{mM}$ DDT (...); all voltammograms are first scans.

six 0.50-mL aliquots. Then known amounts of nitrite standard were successively spiked into each vial followed by appropriate amounts of the Griess assay reagents (i.e. sulfanilamide and NED solutions). The mixtures were incubated for $15 \mathrm{~min}$. The resulting solutions are then diluted to $1.00 \mathrm{~mL}$ with deionized water. Nitrite concentration in each sample was then quantified using known procedures of standard addition method by measuring the absorbance at $540 \mathrm{~nm}$. All results presented, unless otherwise mentioned, are averages of triplicate trials. Results are reported as averages of replicate trials \pm standard deviation

\section{NOS assays}

The second step of the NOS reaction, i.e. the conversion of $\mathrm{N}$ hydroxy-L-arginine (NHA) to L-citrulline and NO, is generally used in NOS mechanistic investigations and was similarly used here to test the performance of various methods in nitrite quantification. The NOS reaction of this step is facilitated by addition of $\mathrm{H}_{2} \mathrm{O}_{2}$. For validation, three different test samples with three different DTT concentrations, $0,0.4$ and $1 \mathrm{mM}$, were prepared in $\mathrm{pH} 7.4$ HEPES buffer, each containing $0.1 \mathrm{mM} \mathrm{NHA}$ and $10 \mu \mathrm{M} \mathrm{H}_{4} \mathrm{~B} .300-\mu \mathrm{L}$ aliquots of each test solution were put in small reaction tubes fitted on a heating block; the tubes were kept $15 \mathrm{~min}$ at $37^{\circ} \mathrm{C} .5 \mu \mathrm{L}$ of purified iNOS-oxygenase domain (iNOSoxy, $22 \mu \mathrm{M}$ ) were then added to the reaction vials. The solutions were kept for another $5 \mathrm{~min}$ at $37^{\circ} \mathrm{C}$. The NOS reaction is started by adding $0.25 \mu \mathrm{L}$ of $50 \mathrm{wt} . \%$ $\mathrm{H}_{2} \mathrm{O}_{2}$ and allowed to proceed for $20 \mathrm{~min}$ at $37^{\circ} \mathrm{C}$. The reactions were quenched by adding $100 \mu \mathrm{L}$ ice-cold deionized water into the reaction vessels and stored in ice for calibration and standard addition measurements. All results presented, unless otherwise mentioned, are averages of triplicate trials. Results are reported as averages of replicate trials \pm standard deviation.

\section{Results and discussions}

\section{Electrochemical results}

In the first step of the Griess assay, sulfanilamide, under acidic conditions, reacts with nitrite to form sulphanilamide-diazonium intermediate. Most diazonium derivatives can be reduced electrochemically within the potential window of +300 to $-500 \mathrm{mV}$ versus $\mathrm{Ag} / \mathrm{AgCl}[23-25]$.

Fig. 1A shows the cyclic voltammogram for the reduction of sulfanilamide-diazonium ion formed in situ. The voltammetric signature in the form of the irreversible reduction peak near $-100 \mathrm{mV}$ shows quantitative formation of the diazonium derivative upon reaction of sulfanilamide with added nitrite under acidic conditions. A number of diazonium derivatives have been shown to form in similar conditions, and can be electrochemically reduced to graft the electrode surface in question [23]. Pure sulfanilamide does not give this electrochemical response. Also, the irreversible reduction wave in Fig. 1A exhibits a general behavior characteristic of the electrochemical behavior of aryl-diazonium derivatives in that the first scan generates highly reactive aryl radicals which attach to the electrode surface and prevent direct electrochemical reduction during subsequent cycles. As a result, and unless the electrode surface is thoroughly cleaned and polished, second and subsequent cycles do not show any reduction (Fig. 1A). This typical behavior is another indicator pointing to the formation of the sulfanilamide diazonium derivative upon nitrite addition.

The diazonium intermediate that forms during the first step of the Griess assay is vulnerable towards most reducing agents, especially dithiothreitol, which is widely used in media for NOS reactions [16,26]. Fig. 1B shows the effect of DTT concentrations on the sulfanilamide-diazonium intermediate. All scans in Fig. 1B are first scans only, after which the electrode is thoroughly cleaned and polished. Increasing the concentration of DTT from 0.5 to $1.0 \mathrm{mM}$ significantly decreases the diazonium reduction current. The addition of a large excess $(>10 \mathrm{mM})$ of DTT destroys completely the diazonium intermediate as evidenced by total disappearance of its reduction peak (results not shown).

\section{UV-vis spectroscopic results}

Diazonium intermediates frequently absorb at or around $375 \mathrm{~nm}$ [19]. Fig. 2A shows the location of the absorption band corresponding to the sulfanilamide-diazonium ion formed in situ upon addition of $0.1 \mathrm{mM}$ nitrite to sulfanilamide; the absorbance was measured at regular time intervals. An absorption band at $365 \mathrm{~nm}$ appears within $5 \mathrm{~min}$ of nitrite addition. The absorption continues to increase steadily through the first $15 \mathrm{~min}$ and then stabilizes as a result of nitrite depletion.

The interaction of DTT with the diazonium intermediate of the Griess assay can also be monitored using absorbance spectroscopy. Addition of $0.5 \mathrm{mM}$ DTT to the diazonium derivative first gives rise to a transient peak that is slightly red-shifted, which then decays over few minutes with an isosbestic point at $406 \mathrm{~nm}$ (Fig. 2B). The transient peak corresponds likely to a preliminary association complex between DTT and the diazonium ion, an association that serves as a preamble to internal electron transfer which then decom- 

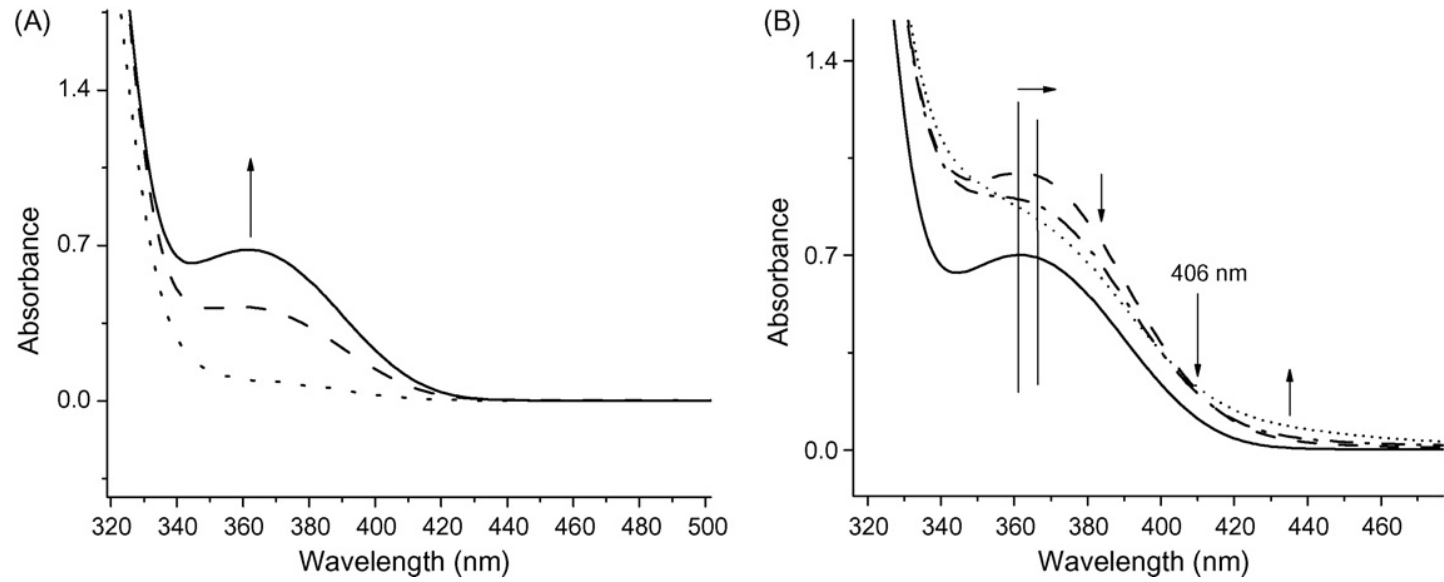

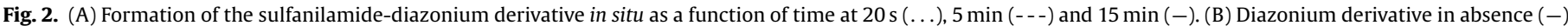

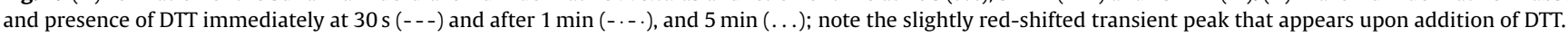

poses the diazonium derivative. The decomposition observed with absorbance spectroscopy is consistent with our electrochemical results, which show decrease of the diazonium derivative upon addition of DTT. The decomposition of the diazonium derivative is the result of electron transfer from the reducing thiol (DTT).

\section{Effect of DTT on the slope of the calibration curve}

Fig. 3 shows how the sensitivity of the Griess assay using external calibration is affected by the presence of increasing amounts of DTT in nitrite standards. The series of curves unequivocally show that as the concentration of DTT increases, a marked decrease in the slope of the linear calibration results. The presence of DTT greatly affects the level of absorptivity at $540 \mathrm{~nm}$ of the final azo-compound produced by the Griess reagents in the presence of nitrite. The decrease in absorptivity is proportional to the concentration of DTT present as shown by the inset in Fig. 3. The effect of DTT on the slope of the Griess calibration curve is rationalized in terms of its effect on the initial cationic diazonium derivative of the two-step assay. However, we need to point out at this point that it is conceivable that DTT, as a dithiol species, may react directly with nitrite to form a mono- and/or di-nitroso-DTT derivatives, which may also contribute to the decreased absorbance of the final azo-compound. To test for this possibility, we have conducted experiments in which we varied the order and the time of addition of DTT (i.e. before or

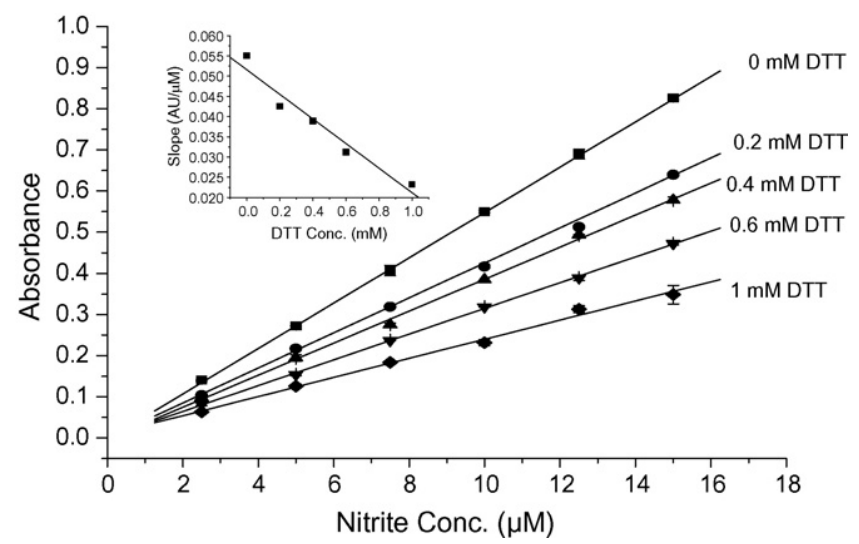

Fig. 3. Effect of DTT concentration on the slope of the calibration curve of the Griess assay. Numbers next to the curves indicate the corresponding DTT concentration in the assay medium; Data points reported as averages \pm standard deviation. Inset: plot of assay sensitivity (absorbance units per $\mu \mathrm{M}$ of nitrite) as a function of DTT concentration. after formation of the cationic intermediate) and comparing final absorbances in both cases, we unequivocally show that this reaction, if any, does not contribute to the loss of the final absorbing compound in the Griess assay (Supporting information, Figure S1). In addition, we show that the reaction of a simpler diazonium cation (para-nitrobenzene diazonium) formed and purified in conditions similar to the first step of the Griess (i.e. reaction of the amine form with nitrite under acidic conditions) takes place quantitatively with added DTT and yields the disulfide (i.e. the oxidized) form (Supporting information, Figure S2).

It is important to note that varying times $(10,15,20 \mathrm{~min})$ of interaction of DTT (with nitrite and with the Griess cationic intermediate) do not change the conclusion of our findings, and always show that the primary interaction of DTT that accounts for the loss of the final azo dye is with the cationic intermediate of the first step of the Griess assay.

These observations are consistent with our electrochemical and spectroscopic results pointing to the reductive decomposition of the initial diazonium-sulfanilamide cation upon addition of the reducing agent DTT. It is important to mention that the concentrations of DTT in NOS reaction media that we used here are typical

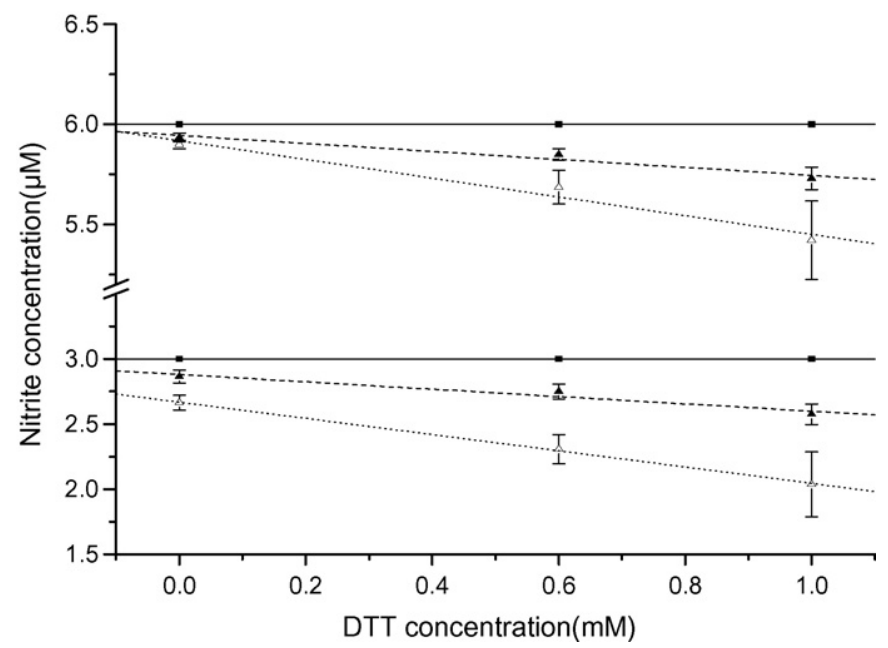

Fig. 4. Comparison of values obtained from calibration and standard addition methods for two levels of nitrite as working "unknowns" in the presence of different DTT concentrations. Expected values for the two concentrations (solid line with solid squares) are to be compared with recovered values using standard addition (dashed line with solid triangles) and calibration (dotted line with open triangles). Data points reported as averages of triplicate trials \pm standard deviation. 
concentrations used in NOS investigations [16,26]. These observations indicate that the response of the Griess assay is compromised as the concentration of DTT increases. This effect is significant with concentrations of DTT as small as $0.2 \mathrm{mM}$ for the micromolar range of nitrite (or nitric oxide) routinely encountered in NOS investigations. In addition, the series shows that the $1-\mathrm{mM}$ concentration level of DTT, usually used in reaction media supporting NOS reactions, depresses the response of the assay by over $44 \%$.

The decrease of the response of the Griess assay as a result of DTT presence in reaction media results in significant discrepancy between true nitrite concentrations and quantities determined from the Griess assay using blind external calibration curves, even when DTT is taken into account. In fact, the concentration of DTT, being a sacrificial reducing agent in the enzyme buffer medium, is generally not known accurately at the time of the assay. In addition, even if the concentration can be estimated, as we show in Fig. 3, the sensitivity of the assay (slope of the linear curve) is seriously depressed at higher DTT concentration which will affect the evaluation of lower nitrite concentrations (or NO in the case of NOS reaction) encountered in experiments at limited enzyme turnovers, where lower nitrite (or nitric oxide) concentrations are encountered. Other differences between media used to construct calibration curves for the Griess assay and the samples matrix also affect the accuracy of the results. For instance, in the case of the NOS reaction, slight differences in the concentration of NADPH, another reducing agent that can interact with the diazonium intermediate [21], also result in noticeable discrepancies in analyte recovery tests. Together, these results show that blind external calibrations yield large errors in the determination of nitrite and, thus, of nitric oxide in the case of NOS reaction, particularly at low concentrations such as those encountered under initial rates settings. The large concentration errors caused by DTT would significantly affect derived kinetic and mechanistic interpretations.

\section{Comparison of the performance for calibration and standard addition methods}

Because the presence of DTT changes the accuracy and sensitivity of the Griess assay, we examined whether the method of standard additions would better reproduce nitrite concentrations compared to the method using external calibration [19].

The calibration method shows significant deviations from actual sample values at 3.0 and $6.0 \mu \mathrm{M}$ levels in the presence of increasing concentrations of DTT. It is important to note that the working solutions of nitrite mixed with DTT are prepared fresh and used on the spot for the calibration and standard additions [27]. The poor analyte recovery is particularly worse for high DTT concentrations and/or low nitrite concentrations (Fig. 4). As for standard addition method, although it does not entirely eliminate the matrix effect, it has a remarkable performance compared to external calibration, especially if one considers the level of DTT interference present in the assay. One of the attributes of the procedure of standard addition, because it uses spiked analyte samples, is the fact that it averages the depressing effect of DTT at various nitrite concentrations, and actually gives weight to the relatively lower effect of DTT observed at higher nitrite concentrations. The resulting observed effect of DTT present in the analyte sample is smaller than in the original non-spiked sample. However, this method, although it decreases the effect of DTT, will not completely eliminate it because there will be always a finite level of reaction of DTT with the diazonium cation generated in the first step of the Griess assay. Methods aiming at eliminating the interference by background thiols in cases such as measurement of S-nitrosothiols in biological samples are known; these include an alkylation step of interfering thiols in the medium by $N$-ethylmaleimide (NEM) during sample preparation $[28,29]$. Conceivably, this alkylation method can also be used here to minimize or eliminate the reducing effect of thiols such as DTT in the Griess assay. However, this extra step is not typically used in Griess assays performed in the context of investigation of NOS enzymatic reactions. The standard additions method as shown here significantly minimizes the DTT interference. Despite a certain level of observed deviations from actual concentrations, the method is still far more reliable than blind external calibration when DTT and other potential interferences are involved. In addition, contrary to matrix-corrected external calibration (i.e. with the attempt to compensate for a known level of DTT), the exact level of DTT in the test solution does not need to be known for standard additions method [19].

\section{Effect of DTT on the determination of NOS turnover using the Griess assay}

In this section we wanted to evaluate the effect of various levels of DTT on actual NOS turnover rates through measurements of NO produced. The catalytic oxidation of $\mathrm{N}$-hydroxy-L-arginine by inducible NOS oxygenase (iNOSoxy) results in nitric oxide production which is measured under the conditions described in Section 2. The NO produced is evaluated through measurement of nitrite
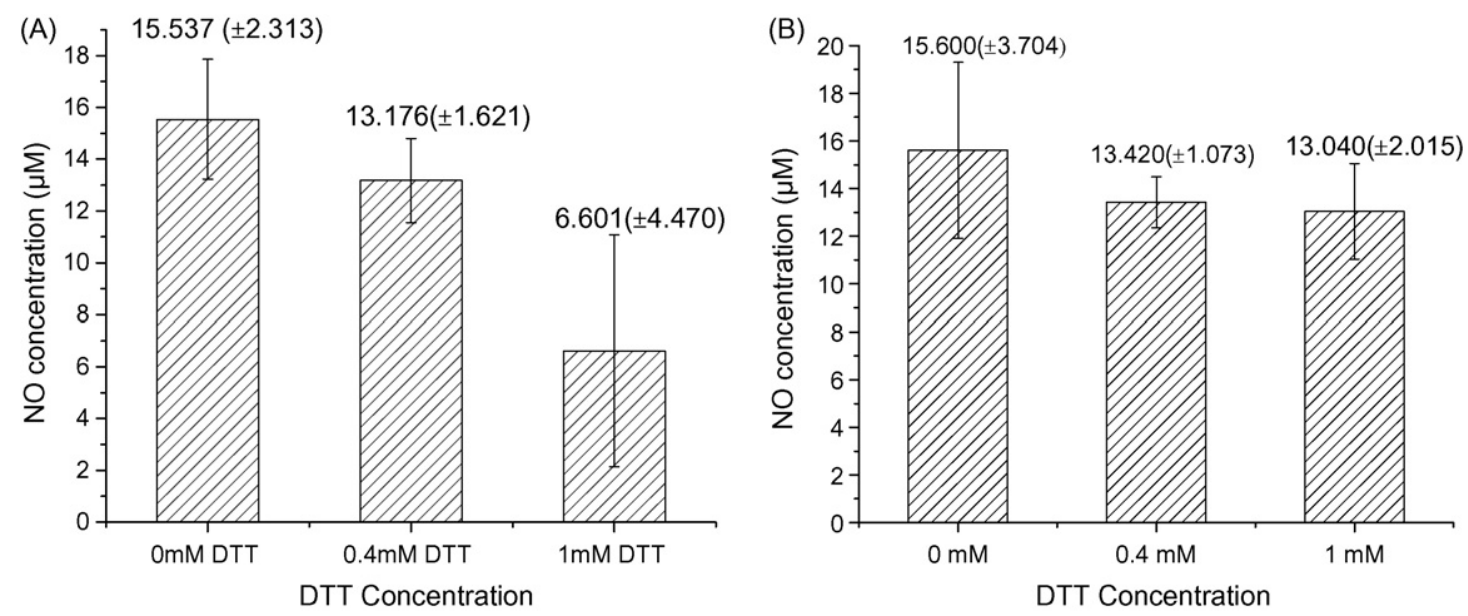

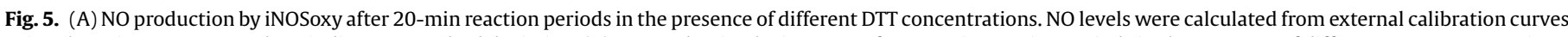

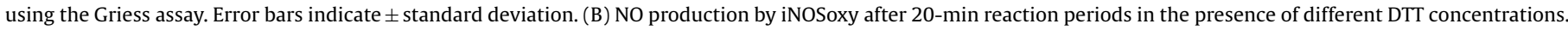
NO levels were measured using standard addition method and the Griess assay. Error bars indicate \pm standard deviation. 
using the Griess assay and suitable calibration curves. Fig. 5A shows that the level of DTT in the enzymatic reaction medium affects seriously the findings of the Griess assay and, thus, NOS turnover determination. The amount of NO measured becomes significantly lower as the concentration of DTT in the NOS reaction medium increases (Fig. 5A).

The increasing levels of DTT transferred from the reaction medium to the assay medium likely contribute to greater degradation of the sulfanilamide diazonium intermediate of the Griess assay, which, in turn, results in lower apparent NO concentrations. In addition to accuracy, the standard deviation of nitric oxide determination is also affected (see error bars on chart, Fig. 5A) at high levels of DTT $(\sim 1 \mathrm{mM})$, where the concentration of the absorbing azo-product of the Griess reaction becomes critically low. Assays of nitric oxide from iNOSoxy reactions containing $0 \mathrm{mM}$ DTT and $0.4 \mathrm{mM}$ DTT resulted in relatively close concentrations of 15.537 $( \pm 2.313) \mu \mathrm{M}$ and $13.176( \pm 1.621) \mu \mathrm{M}$, respectively (for the enzyme amount and reaction time as described in Section 2). This suggests that, at least for this typical reaction setting, the $0.4 \mathrm{mM}$ DTT level does not severely compromise the Griess assay. NOS reaction media with DTT concentration levels of $0.5 \mathrm{mM}$ or higher give extremely low apparent NO concentrations when using external calibration, which can greatly underestimate corresponding NOS turnover rates.

Because the method of standard addition performed much better than the method of external calibration in terms of analyte (NO) recovery (Section 3.4), we examined if this can still hold true when measuring NO produced by NOS in reaction media with various levels of DTT. Fig. 5B shows NO concentrations measured from NOS reaction media with $0.0,0.4$ and $1.0 \mathrm{mM}$ DTT. Here again, the standard addition method shows improved NO recoveries compared to the method of external calibration, even at DTT levels as high as $1.0 \mathrm{mM}$ (Fig. 5B). The standard addition method has been previously shown to correct for the poor performance of external calibration in a multiple-step assay, developed for the determination of banned azo dyes in different leather types [30]. In our case, the standard addition method, due to its inherent use of spiked samples, may be benefiting from a positive contribution of higher concentrations where the DTT effect on the assay is relatively repressed.

\section{Conclusion}

Our electrochemical and spectrophotometric analyses indicate that the vital diazonium intermediate of sulfanilamide in the Griess assay reacts with the reducing agent DTT in solution which causes a decrease in the concentration of the final absorbing azo-compound. Therefore, the presence of DTT and other similar reducing agents significantly affects the measurement of nitrite concentration using the traditional Griess assay and blind external calibration methods. The performance of the method is especially poor at low nitrite (or NO) concentrations. The apparent lower-concentrations observed can compromise the interpretation of initial reaction rates and derived structure-function analysis. Our work shows that the 1$\mathrm{mM}$ concentration level of DTT, often used in reaction media of NOS reactions, depresses the response of the Griess assay by over $44 \%$.
The standard addition method, on the other hand, performs better than the conventional external calibration method, both in analyte recovery studies, and in actual measurements of NO production in NOS catalytic reactions. While the accuracy and sensitivity issues of the Griess assay in the presence of reducing agents are presented here in the context of the NOS reaction and NO determinations, this problem is quite general [21], and applies to all cases in which the diazonium intermediate of the Griess reaction is exposed to reducing agents.

\section{Acknowledgment}

The authors wish to acknowledge financial support by the US DOE-BES through a grant of the "Catalysis Science" program to M.B. (DE-FG02-03ER15462). This research was partially supported by an FRD grant from CSU.

\section{Appendix A. Supplementary data}

Supplementary data associated with this article can be found, in the online version, at doi:10.1016/j.talanta.2008.12.074.

\section{References}

[1] Z. Zhang, D. Naughton, P.G. Winyard, N. Benjamin, D.R. Blake, M.C. Symons, Biochem. Biophys. Res. Commun. 249 (1998) 767-772

[2] W.K. Alderton, C.E. Cooper, R.G. Knowles, Biochem. J. 357 (2001) 593-615.

[3] C.F. Witteveen, J. Giovanelli, M.B. Yim, R. Gachhui, D.J. Stuehr, S. Kaufman, Biochem. Biophys. Res. Commun. 250 (1998) 36-42.

[4] Y. Chen, K. Panda, D.J. Stuehr, Biochemistry 41 (2002) 4618-4625.

[5] M.A. Marletta, A.R. Hurshman, K.M. Rusche, Curr. Opin. Chem. Biol. 2 (1998) 656-663.

[6] K.D. Kroncke, K. Fehsel, V. Kolb-Bachofen, Nitric Oxide 1 (1997) 107-120.

[7] W. Wang, N. Inoue, T. Nakayama, M. Ishii, T. Kato, Anal. Biochem. 227 (1995) 274-280.

[8] T.S. Maurer, H.L. Fung, Nitric Oxide 4 (2000) 372-378.

[9] V.B. Kumar, A.E. Bernardo, M.M. Alshaher, M. Buddhiraju, R. Purushothaman, J.E. Morley, Anal. Biochem. 269 (1999) 17-20.

[10] T.P. Misko, R.J. Schilling, D. Salvemini, W.M. Moore, M.G. Currie, Anal. Biochem. 214 (1993) 11-16.

[11] T.E. Casey, R.H. Hilderman, Nitric Oxide 4 (2000) 67-74.

[12] R.W. Nims, R.A. Lubet, J. Toxicol. Environ. Health 46 (1995) 271-292.

[13] K.M. Miranda, M.G. Espey, D.A. Wink, Nitric Oxide 5 (2001) 62-71.

[14] M.J. Moorcroft, J. Davis, R.G. Compton, Talanta 54 (2001) 785-803.

[15] A. Iannone, A. Tomasi, F. Giovannini, A. Bini, F. Daneri, M. Staffieri, R.R.C. Canali, S. Bergamini, Biol. Chem. 5 (2001) 349-360.

[16] S. Boggs, L. Huang, D.J. Stuehr, Biochemistry 39 (2000) 2332-2339.

[17] T.M. Dawson, V.L. Dawson, Neuroscientist 1 (1995) 7.

[18] D.S. Bredt, S.H. Snyder, Annu. Rev. Biochem. 63 (1994) 175.

[19] P.N. Indika, M. Bayachou, Anal. Bioanal. Chem. 379 (2004) 1055-1061.

[20] C.P. Verdon, B.A. Burton, R.L. Prior, Anal. Biochem. 224 (1995) 502-508.

[21] A. John Moody, F.L. Shaw, Anal. Biochem. 356 (2006) 154-156.

[22] J.B. Fox, F.B. Suhre, Crit. Rev. Anal. Chem. 15 (1985) 283-313.

[23] S. Baranton, D. Belanger, J. Phys. Chem. B 109 (2005) 24401-24410.

[24] S.S.C. Yu, A.J. Downard, e-J. Surf. Sci. Nanotechnol. 3 (2005) 294-298.

[25] E.H. Seymour, N.S. Lawrence, M. Pandurangappa, R.G. Compton, Microchim. Acta 140 (2002) 211-217.

[26] M. Delgado, E.J. Munoz-Elias, R.P. Gomariz, D. Ganea, J. Immunol. 162 (1999) 4685-4696.

[27] We have found that older stock solutions properly stored within the day give essentially the same results. Authors thank a reviewer for pointing to this detail.

[28] E.S. Ng, D. Jourd'heuil, J.M. McCord, D. Hernandez, M. Yasui, D. Knight, P. Kubes, Circ. Res. 94 (2004) 559-565.

[29] R. Marley, M. Feelisch, S. Holt, K. Moore, Free Radic. Res. 32 (2000) 1-9.

[30] L.H. Ahlstrom, S. Amon, L. Mathiasson, J. Sep. Sci. 28 (2005) 2407-2412. 